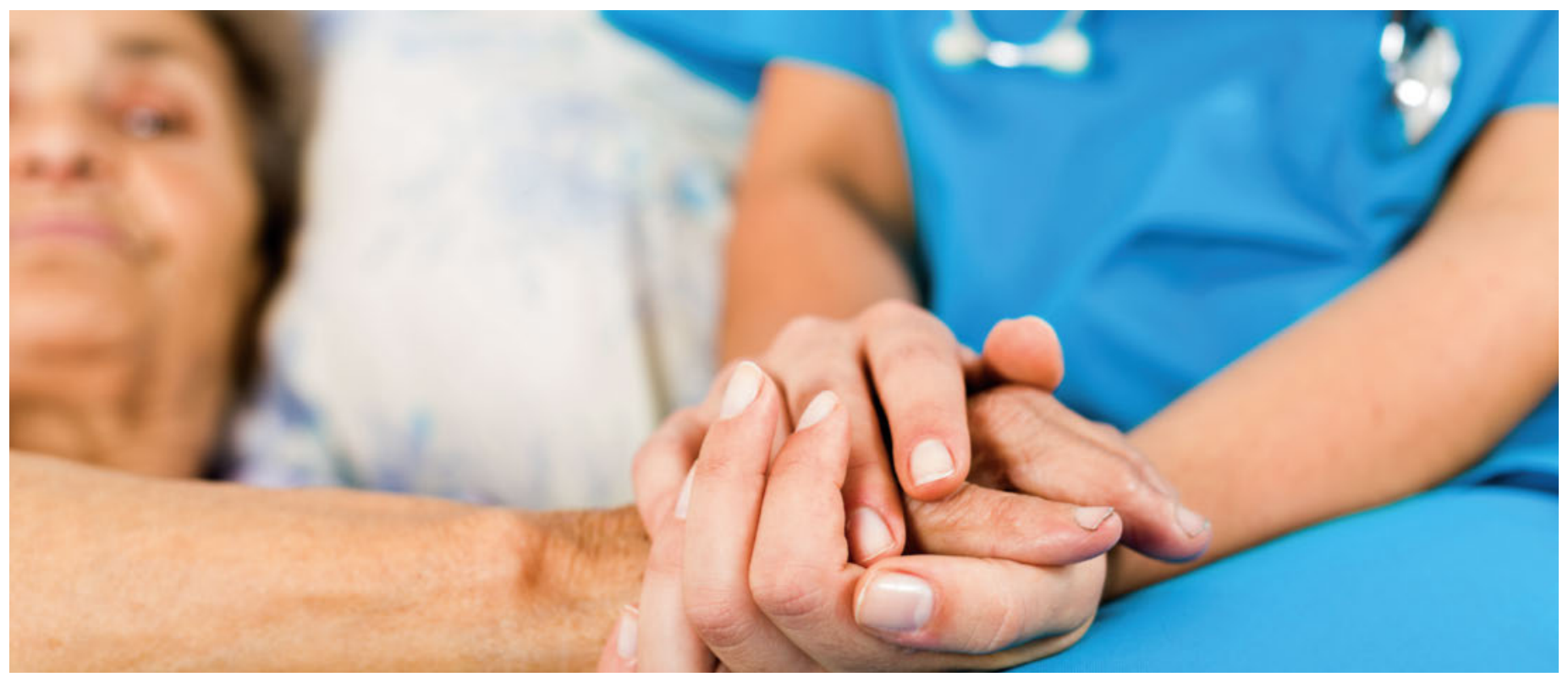

\title{
Prise en charge et traitement des personnes atteintes de démence
}

\section{Georg Bosshard ${ }^{a}$, Michelle Salathéb}

a PD Dr méd., Clinique de gériatrie, Hôpital universitaire de Zurich (Président de la sous-commission), membre de la FMH

b lic. iur., MAE, Secrétaire générale adjointe ASSM

Cf. www.ofsp.admin.ch sous «stratégies nationales en matière de santé»

Les nouvelles directives médico-éthiques «Traitement et prise en charge des personnes atteintes de démence» ont paru et sont disponibles dès à présent en version imprimée. Elles ont été élaborées dans le cadre de la stratégie nationale en matière de démence $2014-2019^{1}$ et en collaboration avec la Société suisse de gérontologie. Les présentes directives s'entendent comme un guide pratique pour les questions éthiques et les situations de conflits dans la prise en charge et le traitement des personnes atteintes de démence.

Le sujet ne laisse pas indifférent: pendant la période de consultation de juin à août 2017, le projet de directives a suscité un vif intérêt; plus de 80 institutions, organisations et personnes se sont exprimées. La sous-commission responsable de l'élaboration des directives, dirigée par PD Dr Georg Bosshard de Zurich, a discuté toutes les remarques et suggestions qui lui sont parvenues pendant la période de consultation et les a intégrées au texte ou a apporté les adaptations correspondantes.
Dans l'ensemble, les réponses étaient très positives. Le texte équilibré, sa pertinence pour la pratique et sa bonne compréhension ont tout particulièrement été soulignés. Le fait que le texte mette trop l'accent sur les aspects médicaux et privilégie une approche pathologique plutôt que compréhensive a fait l'objet de quelques critiques. A cet égard, nous aimerions rappeler que les directives médico-éthiques de l'ASSM s'adressent prioritairement aux professionnels de la santé; la Société suisse de gérontologie prévoit d'élabo- 
rer un complément aux directives à l'attention des professions non médicales.

Parmi les différents thèmes traités dans les directives, les «mesures de contrainte» et le "placement à des fins d'assistance» ont - sans surprise - suscité de nombreuses réactions lors de la procédure de consultation. Les

\section{Pendant la période de consultation de juin à août 2017, le projet de directives a suscité un vif intérêt; plus de 80 avis nous sont parvenus.}

médecins des hôpitaux ont fait part de leur difficulté concernant l'obligation d'informer le représentant légal en cas de recours à des mesures limitatives de mouvements et d'obtenir son consentement pour les mesures de contrainte médicales (administration de médicaments). Le texte définitif a tenu compte de cette critique et a été complété comme suit: «De plus, le consentement du représentant légal est nécessaire, sauf en cas d'urgence et d'interventions de crises temporaires telles qu'elles peuvent se présenter dans le cas de patients déments hospitalisés.»

La recommandation de recourir à l'autorité de protection de l'enfant et de l'adulte (APEA) lorsqu'une personne atteinte de démence s'oppose à son placement dans une institution a donné lieu à des réactions mitigées. On craignait, d'une part, que - sur la base des directives de l'ASSM - des personnes soient placées contre leur volonté, sans l'implication de l'APEA et sans procédure formelle susceptible de recours. D'autre part, il a été souligné que les personnes atteintes de démence n'ont souvent pas conscience de leur maladie et ne consentent que rarement à un placement; si on se base sur les directives, le recours à l'APEA s'imposerait dans presque tous les cas, ce qui n'est considéré ni comme pertinent, ni comme praticable. La version définitive des directives contient des précisions par rapport au projet de texte mis en consultation; les passages ayant fait l'objet de discussions controversées proposent à présent une orientation claire pour la pratique.

Enfin, la nécessité de disposer de ressources structurelles et personnelles suffisantes pour appliquer les directives a également été soulignée dans de nombreuses réponses et doit être comprise comme une sollicitation envers la pratique. Les directives s'adressent

\section{Les directives s'adressent aux médecins,} infirmières et infirmiers et autres thérapeutes.

aux médecins, infirmières et infirmiers et autres thérapeutes. C'est pourquoi elles ne traitent pas de thèmes s'inscrivant à un niveau supérieur - par exemple le développement d'offres de soins conformes aux besoins tout au long de la chaîne d'approvisionnement. Ces thèmes, qui sont approfondis dans d'autres domaines de la stratégie nationale en matière de démence, sont toutefois des conditions préalables indispensables à l'applicabilité des directives.

Crédit photo

(C) Lighthunter | Dreamstime.com

\section{Note}

Les directives médico-éthiques de I'ASSM peuvent être téléchargées en allemand, français, italien et anglais. Les brochures (en allemand et en français) peuvent être commandées gratuitement - même en grande quantité - sous: assm.ch/directives. 